\title{
A psychometric approach to intuitive physics
}

\author{
CEDAR RIENER, DENNIS R. PROFFITT, and TIMOTHY SALTHOUSE \\ University of Virginia, Charlottesville, Virginia
}

\begin{abstract}
The literature on intuitive physics shows that many people exhibit systematic errors when predicting the behavior of simple physical events. Most previous research has attributed these errors to factors specific to a certain class of tasks. In the present study, we investigated the possibility that intuitive physics performance may be related to general measures of cognitive ability. Two hundred four adults (ages, 20-91 years) were presented with five pairs of intuitive physics questions. It was found that performance on the intuitive physics items was moderately intercorrelated, suggesting that they were tapping into a unitary construct. Despite the correlation with factors that decline with advancing age, intuitive physics performance was not correlated with age $(r=.00)$. The findings are discussed in the context of research on intuitive physics, as well as research on cognitive aging.
\end{abstract}

Research into intuitive physics demonstrates that a large percentage of the adult population holds strikingly erroneous views about basic physical principles that govern the motions of objects in the world, a world in which people act and behave quite successfully. For example, when asked to draw the water level on a picture of a tilted glass, about $40 \%$ of young adults draw lines that are not horizontal (McAfee \& Proffitt, 1991). When asked to predict the path that a pendulum bob takes when the string is cut at various points, a large portion of participants make systematically incorrect judgments (Caramazza, McCloskey, \& Green, 1981). The prevalence of these systematic errors among otherwise sophisticated adults has led some investigators to propose that incorrect performance on these tasks was due to specific "naive" beliefs, rather than to a general inability to reason about mechanical systems (McCloskey \& Kohl, 1983). In the present study, we investigated the possibility that performance on intuitive physics tasks also reflects individual differences in cognitive ability.

Research seeking to explain performance on intuitive physics tasks can be grouped into three categories on the basis of the type of hypothesized explanation. First, some have posited explanations for performance that are specific to a certain task or class of tasks. Second, others have focused on factors that are related to general problem complexity or difficulty. A third approach asks whether intuitive physics performance can be explained, in part, by individual differences in cognitive abilities. Although these three approaches are not mutually exclusive, most previous researchers have focused on task-

This research was supported by NSF ITR/Carnegie Mellon Grant 0121629 and ONR Grant N000140110060 to the second author. The authors thank Bobbie Spellman, James Cutting, Timothy Hubbard, and two anonymous reviewers for comments on earlier drafts. Correspondence concerning this article should be addressed to C. Riener, Department of Psychology, University of Virginia, P.O. Box 400400, Charlottesville, VA 22904 (e-mail: criener@virginia.edu). specific and problem complexity explanations. In the present study, we focused on the third approach by relating intuitive physics performance to other cognitive abilities and to age.

By attempting to relate intuitive physics performance to other cognitive abilities across the adult lifespan, this approach offers the potential to both supplement and constrain the conclusions of previous research. Our first step was to determine whether a significant amount of variation in performance on intuitive physics tasks could be explained by a single individual-difference construct. Is performance on a representative sample of intuitive physics tasks intercorrelated, thereby suggesting that these tasks tap a common disposition? If so, this would suggest that although the specific form that errors take on any given intuitive physics task may seem to be unrelated, the aggregate level of performance on several tasks can be partly explained by individual differences common to these tasks. Answering these questions serves to supplement previous explanations of intuitive physics performance by verifying that they are, indeed, a coherent group of tasks. Such results would also constrain previous conclusions by reminding researchers that despite the task-specific nature of systematic errors, individual differences in cognitive ability do account for some of the variation in performance across all of these tasks. Next, we looked at the relationship between intuitive physics performance and other cognitive abilities across the adult lifespan. In other words, if performance on these intuitive physics tasks is indeed intercorrelated, how does this intuitive physics performance relate to other measures of cognitive ability? These results will help place intuitive physics in the broader contexts of cognitive abilities and of how cognitive abilities may change across the lifespan.

\section{Task-Specific Approaches}

Initial approaches to explaining adult performance on intuitive physics tasks focused on the nature of the errors 
that people make. Guided by pioneering developmental research (e.g., Piaget \& Inhelder, 1963), early investigators of intuitive physics tasks in adults saw the systematicity of errors as symptomatic of incorrect intuitive theories or beliefs held by those participants who exhibited the errors (Kaiser, McCloskey, \& Proffitt, 1986; McCloskey \& Kohl, 1983). This approach to intuitive physics invoked factors specific to a particular task to explain both the fact that errors existed and the particular form that the errors took.

To illustrate, Shanon (1976) suggested that errors on questions regarding falling objects could be attributed to the fact that some people hold an Aristotelian theory (that objects fall with constant velocity), rather than the correct Newtonian theory (that objects accelerate as they fall). McCloskey and Kohl (1983) asked participants to predict the behavior of a ball exiting a $\mathrm{C}$-shaped tube lying flat on a table. Forty percent of their participants incorrectly reported that the ball would continue on a curved path after it had exited the tube. This incorrect prediction implies that a putative curvilinear force had been applied to the ball and that it would gradually dissipate after the ball had left the tube. McCloskey and Kohl suggested that these participants held an intuitive theory similar to medieval impetus theory, which did not discriminate between straight and curvilinear forces and asserted that forces are properties of objects themselves, not of the interactions between objects.

These intuitive theory approaches offered quite compelling explanations for the systematic nature of errors on several intuitive physics tasks. However, further examination revealed that there were limits to this approach. First, each intuitive theory explained performance on a limited class of tasks (e.g., impetus theory applies to the C-shaped tube task, but not to the water level task). By invoking factors specific both to the task and to the people who failed the task, these approaches were quite limited in their scope. Second, if holding an incorrect theory causes errors, adherence to these theories should be a stable and consistent characteristic of an individual. Cooke and Breedin (1994) found evidence that suggested that individuals create implicit theories "on the fly," in order to generate responses to intuitive physics questions. Both the form of the question and the form of the response (multiple choice or freehand drawing) affected the number and type of errors on intuitive physics tasks. This inconsistency suggests that performance on these tasks is neither a simple measure of knowledge of the principles of physics nor a reflection of whether one holds an incorrect intuitive theory. For example, whereas participants may respond that a ball exiting a C-shaped tube follows a curved path, they are far less likely to respond that water exiting a curved hose (lying flat on the ground) will continue to curve (Kaiser, Jonides, \& Alexander, 1986), even though the principle assessed is the same.

More recent experiments in intuitive physics have attempted to address the shortcomings of the intuitive theories approach, while continuing to focus on why individuals fail particular tasks, as well as on the manner in which they fail. Some have suggested that rather than applying comprehensive (albeit incorrect) theories, erroneous participants are misapplying simple conceptual heuristics. For example, in an investigation of the development of beliefs about falling objects, Kaiser and colleagues found that the youngest group of participants (preschool and kindergarten) seemed to be using a situation-specific rule ("all objects fall straight down when dropped") before they developed a more comprehensive account of the behavior of falling bodies (Kaiser, Proffitt, \& McCloskey, 1985). Kozhevnikov and Hegarty (2001) combined an intuitive theory approach with heuristics and argued that participants use impetus beliefs as a default heuristic when other information is unavailable or insufficient. These approaches are similar to an intuitive theory approach in that they postulate differences in belief as a root cause of differences in performance on intuitive physics tasks; however, the explanations they provide are entirely specific to the tasks that they address. A similar approach can be seen in an account of performance on the water level task that invokes perceptual biases to explain incorrect performance (McAfee \& Proffitt, 1991).

\section{The Problem Complexity Approach}

Despite the limited success of these various taskspecific approaches, they leave many aspects of the phenomena unexplained. By asking why certain people got specific problems wrong and seeking to explain the form of these errors, these approaches do not provide a general account of what sorts of problems tend to induce errors and what sorts do not. Furthermore, correct performance is virtually ignored by these accounts. The problem complexity account described by Proffitt and Gilden (1989) complements these task-specific approaches by concentrating on general characteristics that make different problems more or less difficult. This account groups problems into two categories according to the number of physical dimensions of motion that must be combined to solve a problem. Problems involving particle motions require that participants pay attention to only one dimension of motion. Complex problems are defined as those that require the problem solver to combine multiple dimensions of motion in order to arrive at the correct solution. Proffitt and colleagues applied this approach to several cases of dynamical motion. Although this approach does not generalize to all of the intuitive physics tasks, its scope is larger than task-specific approaches. On the other hand, because this account essentially splits problems into easy and hard, it lacks the precision that characterizes the task-specific approaches discussed above. The problem complexity account provides a rough prediction of the proportion of errors to be expected, given a certain problem, but it does not explain the particular form that the errors take or account for which participants answer the problem correctly.

\section{The Psychometric Approach}

In addition to task-specific factors, performance on intuitive physics tasks may reflect individual differences 
in cognitive abilities. Psychologists working with other types of cognitive tasks have sought to supplement empirical investigations by administering many tasks to large samples of participants and analyzing the data with correlational procedures often used by psychometricians. For the purposes of this article, we will refer to this sort of investigation (large sample, many tasks, and correlational analyses) as a psychometric approach. For example, some researchers in the field of cognitive aging often adopt this psychometric approach when looking at how cognitive abilities change over time. Our goal in using this approach is to identify the presence of common variance shared among performance measures of intuitive physics and measures of general cognitive abilities. In doing so, we hope to reach some tentative conclusions with regard to the unity of intuitive physics as a set of tasks and the relation of the tasks (as a group) to individual differences, such as age and cognitive ability.

\section{Research Goals}

Previous accounts of intuitive physics performance indicate that errors may be attributable to different causes, depending either on relevant theories, beliefs, or heuristics or on the complexity of the task. Although many of these accounts are quite compelling, a brief survey of the field shows that there are nearly as many explanations as there are tasks. This plethora of explanations challenges the basic assumption that all of these tasks belong to the same grouping, as implied by the common label intuitive physics. In the present study, we sought to assess whether intuitive physics tasks are assessments of a common underlying ability. In other words, is there an individualdifference construct that accounts for a large portion of the variation in the performance across all of these tasks? Additional questions follow from the first. If intuitive physics performance does reflect a unitary disposition or ability, how does this ability relate to other cognitive abilities? Finally, how does intuitive physics performance relate to age and those abilities that have different age trajectories?

\section{METHOD}

\section{Participants}

A total of 204 adults, 20-91 years of age (mean age $=51, S D=$ 17), were recruited through flyers, newspaper advertisements, and referrals from other participants. The participants were generally high functioning, with an average of over 16 years of education and age-adjusted scores on standardized tests between 0.5 and 1 standard deviation above the means from the nationally representative normative sample (Wechsler, 1997). Most of the participants rated their health in the good-to-excellent range. Additional details about the project and other tasks performed during the sessions are described elsewhere (Salthouse \& Ferrer-Caja, 2003).

\section{Stimuli and Procedure}

Intuitive physics questions. The intuitive physics problems consisted of 10 items for which systematic errors have been demon-
When the canoe on the right is dropped, what will the water level be relative to the level on the left $(x)$ ? (lower, the same, higher)

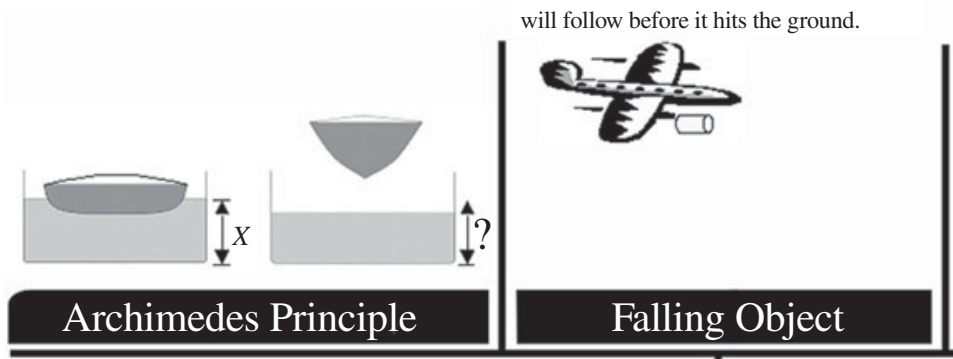

This airplane is carrying a canister as it flies over a field. The plane drops the canister. Draw the path that the canister will follow before it hits the ground.
On the left is a glass of water sitting on the table. On the right, the glass has been tilted.

Assume that the glass and water are now stationery.

Draw the line showing the top surface of the water,

given that it ends at the indicated point.

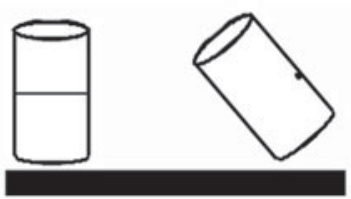

Water Level

\section{C-Shaped Tube}

The $\mathrm{C}$-shaped tube you see below is lying flat on a table (this is an aerial view). A ball is placed in one end and launched so that it proceeds through the tube and out the other side.

Draw the path that the ball will take once it exits the tube, starting at the given point.

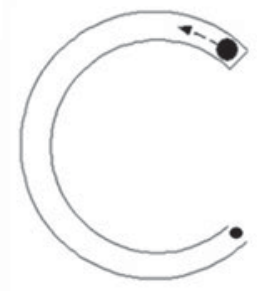

\section{Pendulum}

The pendulum below is swinging from side to side. The string is cut as it reaches the bottom of its swing, swinging in the direction shown (indicated by the scissors). Draw the path of the pendulum bob as it falls to the ground.

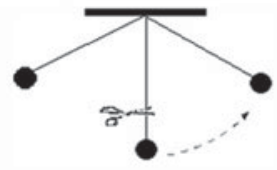

Figure 1. Intuitive physics item examples. 
strated in previous experiments (Caramazza et al., 1981; Kaiser et al., 1985; Kaiser, Proffitt, Whelan, \& Hecht, 1992; McAfee \& Proffitt, 1991; McCloskey, Caramazza, \& Green, 1980). These included two instances of the water level problem, two problems concerning Archimedes' principle, two falling object problems, two pendulum problems, and two C-shaped tube problems (see Figure 1 for examples). We also asked what level of physics courses the participant had completed (none, high school, or college physics courses).

Measures of cognitive abilities. Several standardized measures of cognitive ability were collected. The Vocabulary subtest of the Wechsler Adult Intelligence Scale (Wechsler, 1997) and the Picture Vocabulary subtest of the Woodcock-Johnson Revised test (Woodcock \& Johnson, 1990) were administered to assess acquired knowledge or crystallized ability. The Block Design (Wechsler, 1997) and Analysis-Synthesis (Woodcock \& Johnson, 1990) subtests were used to test cognitive functioning in a manner that minimized the influence of prior knowledge or fluid ability. The Block Design subtest presents participants with a set of blocks with solid or diagonally separated colors on their faces and a pattern that is to be reproduced by arranging the blocks. The Analysis-Synthesis subtest presents participants with components of an incomplete logic puzzle, and they are asked to identify the missing components. These subtests have been identified (McGrew \& Flanagan, 1998) as suitable tests of fluid intelligence, as originally conceived by Horn and Cattell (1967).

\section{RESULTS}

Each of the intuitive physics items was scored as correct or incorrect. A sum was calculated by coding the incorrect responses 0 and the correct responses 1 . For some items (such as the water level problem) scoring was based on a criterion of within $\pm 5^{\circ}$ of the correct answer in a drawing. ${ }^{1}$

Each of the intuitive physics items was moderately correlated with all of the other intuitive physics items, and the internal consistency (coefficient alpha) for the 10 items was .67. This moderate level of internal consistency suggests that all of the intuitive physics items measure a unitary dimension of individual differences.

Once we determined the merit of considering intuitive physics performance as a unitary disposition, we examined how intuitive physics performance in sum was related to the other variables that were collected. The intuitive physics literature has documented that performance on some intuitive physics items is affected by gender (males perform better; Robert, 1990), education (McCloskey \& Kohl, 1983), and physics education (Kaiser,

Fluid Intelligence vs. Chronological Age
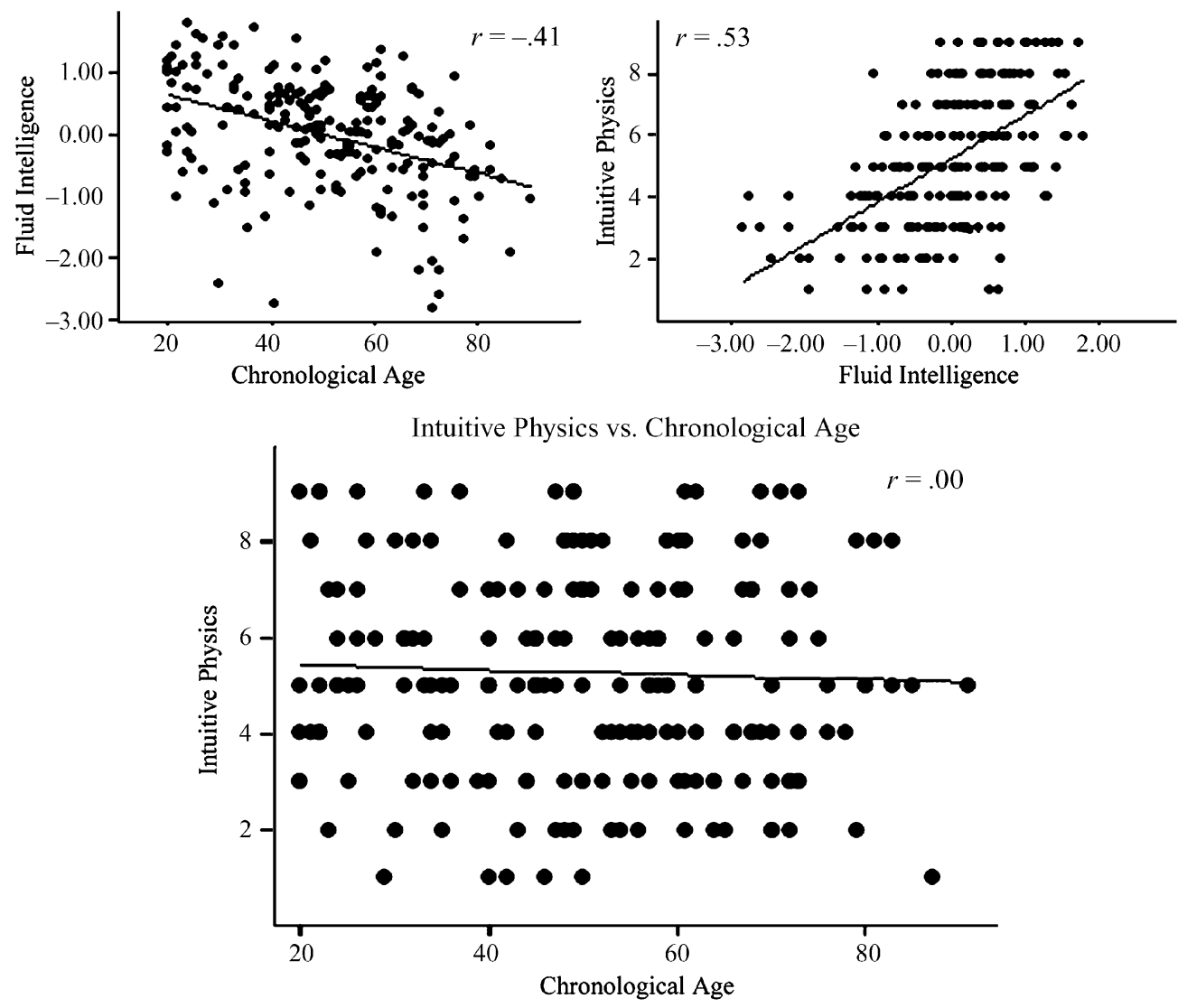

Figure 2. Relationship between age, fluid intelligence, and intuitive physics. 
Jonides, \& Alexander, 1986). Our summary intuitive physics performance measure also showed these effects. Men significantly outperformed women $(\mathrm{M}=0, \mathrm{~F}=1$; $r=-.51$ ). Both of our measures of fluid and crystallized cognitive ability were found to be correlated with intuitive physics performance $(g F, r=.53 ; g C, r=.44)$. Education (number of years of formal education completed, ranging from 11 to 22) and number of physics courses ( 0 for none, 1 for high school, 2 for college, and 3 for advanced college) were also correlated with the intuitive physics score (years of education, $r=.31$; number of physics courses, $r=.49$ ).

The interaction of age and sex was examined as a cross-product term in a hierarchical regression after the effect of both age and sex had been analyzed. Robert (1990) advanced the possibility that the gender difference on the water level task might change across different age categories. However, the cross-product was associated with a nonsignificant ( $p>.75$ ) increment in $R^{2}$ of .00 , suggesting that the male advantage was constant across the adult age range.

The scatterplots in Figure 2 portray the pairwise relations among age, fluid intelligence, and intuitive physics performance. The panel in the upper left illustrates the well-documented negative relation between age and fluid intelligence, and the panel in the upper right illustrates that fluid intelligence and intuitive physics performance were positively correlated. These two relations led to an expectation that increased age should be associated with lower intuitive physics performance, but the bottom panel indicates that this expectation was not confirmed.

In an attempt to understand the surprising absence of a negative relation between age and intuitive physics performance, the data were examined in the context of the path analysis model illustrated in Figure 3. A path analysis serves to tie together relations among multiple variables and is valuable in decomposing correlations among variables. The numbers on the arrows in the figure correspond to standardized partial regression coefficients, which represent the effect of one variable on the other after controlling for other variables in the model. By controlling

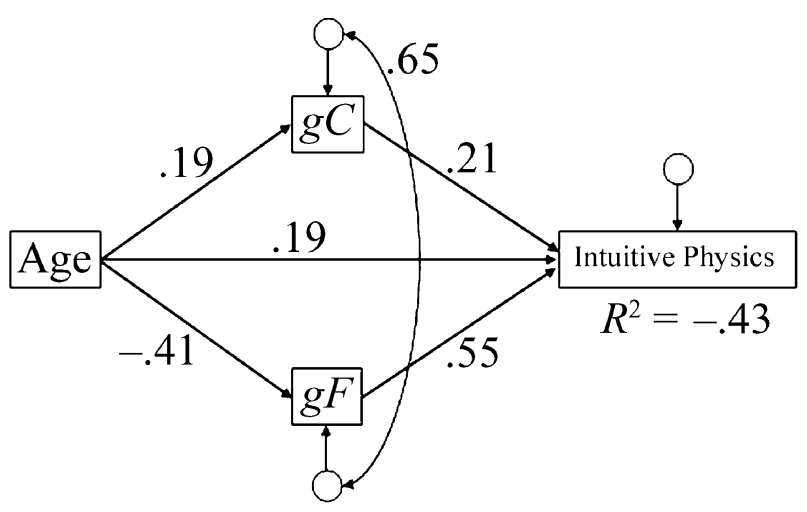

Figure 3. Intuitive physics path analysis. for the other variables, these coefficients offer an estimate of the unique influence of one variable on the other. The direction of the arrows represents a hypothesis about the causal direction of influence (e.g., one would say that age influences $g F$, not vice versa).

The most interesting results of the path analysis are that, in addition to the indirect effects of age through the $g C$ and $g F$ variables, there was a significant direct effect of age on intuitive physics performance. This relation implies that there was no overall relation between age and intuitive physics performance because increased age was associated with an increase in something besides $g C$ that contributed to better performance in the intuitive physics problems.

\section{DISCUSSION}

Performance on the intuitive physics items was moderately intercorrelated, suggesting that a single individualdifference construct may explain some variation in this performance. Several implications stem from this finding. Given the correlations we observed, it is apparent that task-specific approaches cannot fully account for the overall levels of correct performance. Rather, these accounts predict the form that errors are likely to take and attempt to describe the perceptual or reasoning biases that may be responsible for them. Unlike the previous approaches, we do not seek to explain the systematic nature of the errors, just their frequency. In this way, our approach is similar to that of Proffitt and Gilden (1989) but indicates that their approach needs to be revised and elaborated.

The relation between intuitive physics performance and age is surprising, due to the expectation informed by two sources. First, intuitive physics problems resemble traditional mechanical reasoning problems and, thus, would be expected to be related to fluid reasoning ability. Fluid reasoning abilities of all kinds decline with age (Salthouse, 2004); therefore, intuitive physics would also be expected to decline with age. The second source of the expectation is the correlations we observed in the present study. Fluid ability and intuitive physics are highly correlated, even in the context of other variables, such as age and crystallized ability. In our sample, fluid ability declined with increasing age, and thus, on the basis of this result, we would expect performance on intuitive physics tasks to decline with increasing age. Contrary to these expectations, intuitive physics performance remained stable across the lifespan (see Figure 2).

Although they are only speculative at this point, we offer two possible explanations for this paradox. The first is that intuitive physics performance may actually be influenced by some crystallized ability distinct from that measured by vocabulary (and observed in this investigation). This form of crystallized ability could be related to fluid ability in the manner that it is acquired but could be maintained despite advancing age. In order to ascertain whether this is true, more measures of crys- 
tallized ability, perhaps related to knowledge of mechanics and basic physical principles, could be gathered and entered into the path analysis. The second possibility is that there may be several ways of solving these problems, and older adults may compensate for their lower fluid ability by relying on other routes to success. However, the challenge in this case would be to obtain direct evidence of the compensatory mechanism, rather than merely inferring it from other relations. Such compensation is relatively rare in the cognitive aging literature, and this would thus be of interest to that community, if true.

This research has several implications for both the intuitive physics literature and the aging literature. Looking back on the intuitive physics literature, we suggest that what is so intriguing about these studies is the fact that erroneous answers are systematic. Errors are not randomly distributed but, rather, seem to reflect consistent, inherent biases. The present study, however, suggests that the likelihood that someone is going to provide an erroneous answer is related to a general ability that transcends the task-specific intuitive theories that have been proposed to account for the systematicity of errors.

Finally, our research illustrates the advantages to integrating task-specific and task-independent approaches. For the case of intuitive physics, incorporating these two accounts allows for a more comprehensive description of the phenomena. Proffitt and Gilden's (1989) taskindependent account of problem complexity predicts normative levels of performance, whereas task-specific accounts describe the individual differences that prescribe the form that systematic errors are likely to take. Our approach suggests not only that mechanical problems vary in their difficulty (as Proffitt \& Gilden pointed out), but also that people differ in their ability to solve them. This ability seems to be strongly related to both fluid and crystallized abilities and yet, for reasons that are not yet understood, is unaffected by age.

\section{REFERENCES}

Caramazza, A., McCloskey, M., \& Green, B. (1981). Naive beliefs in "sophisticated" subjects: Misconceptions about trajectories of objects. Cognition, 9, 117-123.

Cooke, N. J., \& BREEDIN, S. D. (1994). Constructing naive theories of motion on the fly. Memory \& Cognition, 22, 474-493.

HoRn, J. L., \& CATtELL, R. B. (1967). Age differences in fluid and crystallized intelligence. Acta Psychologica, 26, 107-129.

Kaiser, M. K., Jonides, J., \& AleXANDER, J. (1986). Intuitive reason- ing about abstract and familiar physics problems. Memory \& Cognition, 14, 308-312.

KaIser, M. K., McCloskey, M., \& Proffitt, D. R. (1986). Development of intuitive theories of motion: Curvilinear motion in the absence of external forces. Developmental Psychology, 22, 67-71.

Kaiser, M. K., Proffitt, D. R., \& McCloskey, M. (1985). The development of beliefs about falling objects. Perception \& Psychophysics, 38, 533-539.

Kaiser, M. K., Proffitt, D. R., Whelan, S. M., \& Hecht, H. (1992). Influence of animation on dynamical judgments. Journal of Experimental Psychology: Human Perception \& Performance, 18, 669-690.

KozheVniKov, M., \& HegarTY, M. (2001). Impetus beliefs as default heuristics: Dissociation between explicit and implicit knowledge about motion. Psychonomic Bulletin \& Review, 8, 439-453.

LiBEN, L. S. (1991). The Piagetian water-level task: Looking beneath the surface. In R. Vasta (Ed.), Annals of child development (Vol. 8, pp. 81-143). Greenwich, CT: JAI Press.

McAfee, E. A., \& Proffitt, D. R. (1991). Understanding the surface orientation of liquids. Cognitive Psychology, 23, 483-514.

McCloskey, M., Caramazza, A., \& Green, B. (1980). Curvilinear motion in the absence of external forces: Naive beliefs about the motion of objects. Science, 210, 1139-1141.

McCloskey, M., \& KoHL, D. (1983). Naive physics: The curvilinear impetus principle and its role in interactions with moving objects. Journal of Experimental Psychology: Learning, Memory, \& Cognition, 9, 146-156.

McGrew, K. S., \& Flanagan, D. P. (1998). The intelligence test desk reference (ITDR): Gf-Gc cross-battery assessment. Boston: Allyn \& Bacon.

Piaget, J., \& Inhelder, B. (1963). Intellectual operations and their development. Traite de Psychologie Experimentale, 7, 111-155.

Proffitt, D. R., \& GiLdEN, D. L. (1989). Understanding natural dynamics. Journal of Experimental Psychology: Human Perception \& Performance, 15, 384-393.

RoBERT, M. (1990). Sex-typing of the water-level task: There is more than meets the eye. International Journal of Psychology, 25, 475-490.

Salthouse, T. A. (2004). What and when of cognitive aging. Current Directions in Psychological Science, 13, 140-144.

Salthouse, T. A., \& Ferrer-CaJa, E. (2003). What needs to be explained to account for age-related effects on multiple cognitive variables? Psychology \& Aging, 18, 91-110.

Shanon, B. (1976). Aristotelianism, Newtonianism and the physics of the layman. Perception, 5, 241-243.

WeChSLER, D. (1997). Wechsler Adult Intelligence Scale (3rd ed). San Antonio, TX: Psychological Corporation.

Woodcock, W. R., \& Johnson, M. B. (1990). Woodcock-Johnson PsychoEducational Battery-Revised. Allen, TX: DLM Teaching Resources.

\section{NOTE}

1. This is an accepted convention in the literature of the water level task (Liben, 1991).

(Manuscript received July 21, 2004; revision accepted for publication September 27, 2004.) 